\title{
Carbon Nanotube-adsorbed Polystyrene and Poly(methyl
}

\section{methacrylate) Microspheres}

\author{
Hyoung-Joon Jin ${ }^{1 *}$, Hyoung Jin Choi ${ }^{1}$, Seok Ho Yoon ${ }^{1}$, Seung Jun Myung ${ }^{1}$, Sang Eun Shim ${ }^{2}$ \\ ${ }^{1}$ Department of Polymer Science and Engineering, ${ }^{2}$ Department of Chemical Engineering, Inha \\ University, Incheon 402-751, Korea
}

Phone: +82-32-860-7483, Fax.: +82-32-865-5178, E-mail: hjjin@inha.ac.kr

\section{Experimental Sections}

Materials. Styrene (Junsei Chemicals, Japan) and methyl methacrylate (Aldrich, USA) were distilled under reduced pressure and stored in a refrigerator prior to use. Poly( $N$-vinylpyrrolidone) (PVP-40T; weight-average molecular weight $=40,000$; Sigma Chemical Co.) was used. Ethanol (Samchun Chemical Co., Korea) was used as the reaction medium. The 2,2-azobisisobutyronitrile (analytical grade, AIBN; Junsei) was used as received.

Preparation of PS Microspheres. PS microspheres, $3.0 \mu \mathrm{m}$ in number-average diameter, were synthesized with 1.0 and $1.5 \mathrm{wt} \%$ of AIBN using a well-established dispersion polymerization method in ethanol medium for $24 \mathrm{hrs}$ at $70{ }^{\circ} \mathrm{C}$. The amount of PVP as a steric stabilizer was fixed at $12 \mathrm{wt} \%$ relative to styrene. The polymerization was carried out in a $500 \mathrm{~mL}$ three-neck reaction vessel at a constant stirring speed of $150 \mathrm{rpm}$ under a nitrogen atmosphere. After completing the polymerization, 
the resulting mixture was repeatedly rinsed with methanol and water, which was followed by a final fine PS microspheres being obtained by a filtration process (Figure 1 (a)).

Preparation of PMMA Microspheres. PMMA microparticles were prepared via a dispersion polymerization method, which is one of the most popular methods for making monodispersed polymer particles. The purified methyl methacrylate monomer and a radical initiator (azobisisobutyronitrile) were dissolved at room temperature in methanol containing poly( $N$-vinyl pyrrolidone) as a stabilizer. After removing the oxygen by nitrogen purging, the reaction mixture was heated to $55{ }^{\circ} \mathrm{C}$ and maintained for $24 \mathrm{~h}$. The monodisperse particles obtained were washed by methanol and then dispersed in water. The PMMA particles prepared at $55^{\circ} \mathrm{C}$ had a narrow size distribution with a particle size of $6.5 \mu \mathrm{m}$ (Figure 1 (b)).

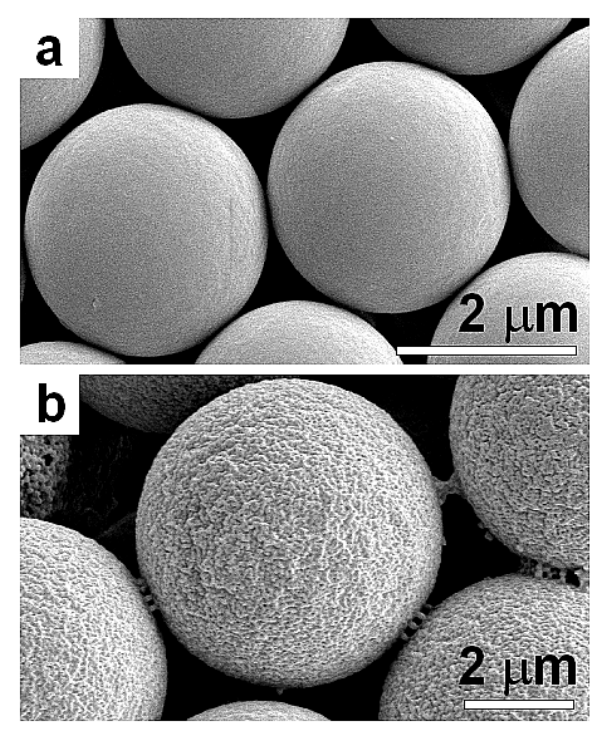

Figure 1. Field-emission scanning electron microscopy (FESEM) images of the (a) polystyrene and (b) poly(methylmetacrylate) seed microspheres.

Purification of Cabon Nanotubes. The multi-walled MWNT (Iljin Nanotech Co., Korea) were synthesized by a thermal chemical vapor deposition (CVD) method. The purity of the pristine MWNT, as received, was $97 \%$. In order to eliminate impurities in the MWNT (such as metallic catalysts), the 
MWNT were treated in $3 \mathrm{M} \mathrm{HNO}_{3}$ at $60{ }^{\circ} \mathrm{C}$ for $12 \mathrm{~h}$, followed by a reflux process in $5 \mathrm{M} \mathrm{HCl}$ at $120{ }^{\circ} \mathrm{C}$ for $6 \mathrm{~h}$. The purity of the acid-treated MWNT was measured to be $99 \%$ using thermogravimetric analysis (TGA, Polymer Lab., TGA1000, UK).

Preparation of Carbon Nanotube Dispersion. The purified MWNTs were dispersed in pure water $(0.5 \mathrm{mg} / \mathrm{mL}$ ) with surfactants such as TX 100 (non-ionic), SDS (anionic), CTAB (cationic) and NaDDBs (anionic) (0.3 wt\% in water). An ultrasound was then applied to the MWNT dispersion using an ultrasonic generator (Kyungill Ultrasonic Co., Korea), which has a nominal frequency of $28 \mathrm{kHz}$, with power of $600 \mathrm{~W}$ for $7 \mathrm{~h}$ at $25 \square$.

Characterizations. The surface morphology and the microsphere shape were observed using a field emission scanning electron microscopy (FESEM, S-4300, Hitachi, Japan). The FESEM images were obtained by collecting the microspheres on an aluminum SEM disk, which was then coated with platinum. The acceleration voltages and the working distances for each image were $15 \mathrm{kV}$ and $6 \mathrm{~mm}$, respectively. the average diameter of the PS and PMMA microspheres were determined using Scion Image ${ }^{\circledR}$ Analyzer Software by counting 100 individual particles from the electronic SEM images.

Electrical Conductivity of Carbon Nanotubes-adsorbed Microspheres. The electric conductivity of the pressed disk-type CNT-coated polymer particles were measured by a four-probe method using a picoammeter with an internal voltage source (487, Keithley, USA) and an impedance analyzer (4284A, HP, USA), respectively.

Electrorheological Characterization. The nanotubes-adsorbed PMMA microspheres were washed several times in pure water to extract the remaining surfactants and dried in a vacuum oven at room temperature. The ER fluids were finally prepared using the dried nanotubes-adsorbed microspheres dispersed in silicone oil (10 vol \%) by sonication. No stabilizers were added to the nanotubes-adsorbed microspheres dispersion in silicone oil. A DC high voltage source was used to apply a voltage to the 
sample. The gap between the two parallel electrodes was fixed precisely at $350 \mu \mathrm{m}$. The microstructure image of the ER fluid was obtained by optical microscopy (BX51, Olympus, Japan). 